\title{
The influence of in vitro pectin
} fermentation on the human fecal microbiome

\author{
So-Jung Bang ${ }^{1 \dagger}$, Gayoung Kim ${ }^{1 \dagger}$, Mi Young Lim², Eun-Ji Song ${ }^{2,3}$, Dong-Hyun Jung ${ }^{1}$, Jun-Seok Kum ${ }^{4}$, \\ Young-Do Nam ${ }^{2,3}$, Cheon-Seok Park ${ }^{1 *}$ and Dong-Ho Seo ${ }^{2^{*}}$ (1)
}

\begin{abstract}
Pectin is a complex dietary fiber and a prebiotic. To investigate pectin-induced changes in the gut microbiome and their effects on the short chain fatty acids (SCFAs) production, we performed in vitro pectin fermentation using the feces of three Korean donors. The pectin degradations in all three donors were observed. While the donors displayed differences in baseline gut microbiota composition, commonly increased bacteria after pectin fermentation included Lachnospira, Dorea, Clostridium, and Sutterella. Regarding SCFAs, acetate levels rapidly increased with incubation with pectin, and butyrate levels also increased after $6 \mathrm{~h}$ of incubation. The results suggest that pectin fermentation increases bacterial species belonging to Clostridium cluster XIV (Lachnospira, Dorea, and Clostridium), with Lachnospira displaying the greatest increase. The results also confirm that pectin fermentation leads to the production of acetate and butyrate.
\end{abstract}

Keywords: Pectin, Fecal microbiota, Fermentation, Short chain fatty acids, Prebiotic

\section{Introduction}

Recent studies have demonstrated that the gut microbiota plays important roles in human health, and are associated with diseases. The pathogenic mechanisms of various diseases and disorders, such as irritable bowel syndrome (Hyland et al. 2014), Crohn's disease (Gevers et al. 2014), and non-alcoholic steatohepatitis/non-alcoholic fatty liver disease (Icaza-Chávez 2013; Sánchez et al. 2017) are associated with the composition and diversity of the gut microbiota (Laparra and Sanz 2010). The constitution of the gut microbiota can be influenced by endogenous and environmental factors, such as one's dietary, antibiotic, xenobiotic, and probiotic intakes (Falony et al. 2016).

Prebiotic food ingredients feed the intestinal microbiota, and can be used to selectively promote the growth of specific microbiota (Laparra and Sanz 2010). Most

\footnotetext{
*Correspondence: cspark@khu.ac.kr; sdh83@kfri.re.kr

${ }^{\dagger}$ So-Jung Bang and Gayoung Kim contributed equally to this work ${ }^{1}$ Graduate School of Biotechnology and Institute of Life Science and Resources, Kyung Hee University, Yongin 17104, Republic of Korea

${ }^{2}$ Research Group of Healthcare, Korea Food Research Institute, Wanju 55365, Republic of Korea

Full list of author information is available at the end of the article
}

prebiotics are carbohydrates, such as inulin, fructooligosaccharide, and human milk oligosaccharide, which are not digested by human digestive enzymes (Bindels et al. 2015; Coppa et al. 2006). Microorganisms in the intestines produce energy and short-chain fatty acids (SCFAs) including acetate, propionate, and butyrate, affecting the host though prebiotic fermentation (Flint et al. 2012). The SCFAs produced by the gut microbiota have positive effects on immune function and ameliorate metabolic diseases such as obesity and type 2 diabetes (Clemente et al. 2012; Gerritsen et al. 2011).

Differences in intestinal microbiota composition can exist depending on diet (Conlon and Bird 2014; David et al. 2014), and in particular, on the types of nutrients ingested in various countries, environments, and cultures (Marques et al. 2010). Asian diets tend to be high in carbohydrates, while western country diets are relatively high in fat (LeCroy and Stevens 2017; Li et al. 2017). The Korean diet, one of the Asian diets, tends to be a highvegetable diet compared with the typical western diet because of traditional foods including Kimchi (Kim et al. 2016; Song and Joung 2012; Lee et al. 2002).

Pectin is a complex polysaccharide found in the cell walls of a variety of vegetables and fruits, which is mainly 
composed of D-galacturonic acid (GalA) with $\alpha-(1-4)$ glycosidic linkages (Sriamornsak 2003). Pectin is a candidate prebiotic because it is not well degraded by human digestive enzymes, but is by microorganisms (Holloway et al. 1983). Pectin is degraded by the gut microbiota, producing SCFAs and changing the composition of the intestinal microbiota (Chung et al. 2016; Marounek and Dušková 1999). Although many studies have confirmed the degradation of pectin and the production of SCFAs by gut microbiota species of the genera Bifidobacterium, Faecalibacterium, Anaerostipes, and Roseburia (Duncan et al. 2004), very few metagenomics studies have been performed on the human gut microbiota following in vitro fermentation of pectin. In addition, no studies have been performed examining pectin utilization and gut microbiota changes in Koreans, which have high vegetable consumption. Thus, in this study, we explored the process of pectin degradation and the compositional changes in the gut microbiota of three Korean subjects after pectin fermentation.

\section{Materials and methods Donor information}

All donors (males, ages 29, 30, and 30) were healthy and did not have any gastrointestinal disease. Donors had consumed a regular diet and had not received antibiotic treatment in the last 6 months. The study was approved by the Institutional Review Board of Kyung Hee University (IRB file no. KHSIRB-17-004). All experiments were performed in accordance with relevant guidelines and regulations. Informed consent was obtained from all participants.

\section{Fecal collection}

Fecal samples $(20 \mathrm{~g})$ were collected from three volunteers under anaerobic conditions and transported within $1 \mathrm{~h}$ after excretion. Fecal samples were diluted fivefold with sterile phosphate-buffered saline in an anaerobic chamber. After mixing, the resultant fecal slurries were homogenized and immediately inoculated in the prepared medium.

\section{Growth media}

Pectin from citrus peel was purchased from SigmaAldrich (St. Louis, MO, USA), and was composed of $>74 \%$ galacturonic acid. Because a basal medium is non-selective and supports the growth of several organisms, we selected a basal medium for this study; the basal medium used was the chopped meat $(\mathrm{CM})$ broth containing $15 \%(\mathrm{v} / \mathrm{v})$ of bovine rumen fluid (CMR). Each liter of CMR consisted of peptone (30.0 g), yeast extract $(5.0 \mathrm{~g})$, dipotassium phosphate $(5.0 \mathrm{~g})$, L-cysteine $(0.5 \mathrm{~g})$, and resazurin $(0.001 \mathrm{~g})$. Pectin was added to a final concentration of $1 \%$. CMR medium with $1 \%$ pectin (CMRP) was stirred on a hot plate to dissolve the pectin, transferred to serum vials, and capped. Sealed CMRP medium was flushed with $99.5 \% \mathrm{CO}_{2}$ gas and sterilized by autoclave at $121^{\circ} \mathrm{C}$ for $15 \mathrm{~min}$.

\section{Batch culture incubations}

To investigate changes in microbial diversity and pectin degradability, $100 \mu \mathrm{L}$ of prepared feces was inoculated into $20 \mathrm{~mL}$ of CMRP medium (baseline). Cultures were incubated at $37^{\circ} \mathrm{C}$ at $150 \mathrm{rpm}$, with sampling at various incubation times $(0,6,12,18,24,36$, and $48 \mathrm{~h})$. All samples were immediately frozen and stored at $-72{ }^{\circ} \mathrm{C}$. Incubations were performed in CMRP medium in triplicate.

\section{Determination of total sugar}

The total sugar in culture was measured by the phenolsulfuric acid method. A $5 \%$ phenol solution $(200 \mu \mathrm{L})$ was added to $100 \mu \mathrm{L}$ of culture supernatant from each time point. The reaction mixture was mixed with $800 \mu \mathrm{L}$ of $99 \%$ sulfuric acid and vortexed. After cooling for $20 \mathrm{~min}$ at $25{ }^{\circ} \mathrm{C}, 250 \mu \mathrm{L}$ of each mixture was added to a 96-well microplate. The absorbance of the phenol-sulfuric acid was measured at $550 \mathrm{~nm}$ using an iMark Microplate Absorbance Reader (BioRad Laboratories, Inc., Hercules, CA, USA).

\section{Determination of reducing sugar}

The reducing sugar in culture was measured using 3,5-dinitrosalicylic acid (DNS). Culture supernatants from each time point $(20 \mu \mathrm{L})$ were diluted in $80 \mu \mathrm{L}$ deionized water. Reducing sugar was detected by adding $300 \mu \mathrm{L}$ of DNS solution and boiling for $5 \mathrm{~min}$. After cooling on ice, the absorbance was measured at $555 \mathrm{~nm}$ using an iMark Microplate Absorbance Reader.

\section{Thin liquid chromatography (TLC) analysis}

TLC analysis was performed with TLC Silica gel 60G $\mathrm{F}_{254} 25$ Glass plates (Merk Millipore, Billerica, MA, USA) after activating at $110{ }^{\circ} \mathrm{C}$ for $5 \mathrm{~min}$. Culture supernatants from each time point were loaded onto TLC plates and placed in a TLC chamber containing a 5:2:3 (v/v/v) solvent mixture of 1-butanol:acetic acid:water for degradation product analysis. Plates were dried, rapidly soaked into $0.3 \%(\mathrm{w} / \mathrm{v}) 1$-naphthol and $5 \%(\mathrm{v} / \mathrm{v})$ sulfuric acid in methanol, dried again, and placed on a $110{ }^{\circ} \mathrm{C}$ oven for $10 \mathrm{~min}$.

\section{DNA extraction and 16S rRNA gene sequencing}

Fecal bacterial DNA from samples taken at various times $(0,6,12$, and $18 \mathrm{~h})$ was extracted using a QIAamp DNA Stool Mini kit (Qiagen, Valencia, CA, USA), in accordance with the manufacturer's instructions, including 
bead-beating twice for $5 \mathrm{~min}$. The V1-V2 regions of $16 \mathrm{~S}$ rRNA genes were amplified by polymerase chain reaction (PCR) using universal primers (8F and 338R) with barcode sequences for multiplexing sample reads. PCR was performed using a PCR Thermal Cycler Dice (Takara, Shuzu, Japan) and recombinant Taq DNA polymerase (Takara). The PCR conditions were as follows: $95^{\circ} \mathrm{C}$ for $5 \mathrm{~min} ; 30$ cycles of $30 \mathrm{~s}$ at $95^{\circ} \mathrm{C}, 1 \mathrm{~min}$ at $61{ }^{\circ} \mathrm{C}$, and $40 \mathrm{~s}$ at $72{ }^{\circ} \mathrm{C}$; and $5 \mathrm{~min}$ at $72{ }^{\circ} \mathrm{C}$. The amplified $16 \mathrm{~S}$ rRNA gene products were purified with an AccuPrep PCR Purification Kit (BIONEER, Daejeon, Korea).

PCR product concentrations were measured on a NanoDrop ND-1000 (NanoDrop Technologies Inc., Wilmington, DE, USA) and mixed to a constant concentration such that the total concentration was $1 \mathrm{mg}$. The Ion Xpress Plus Fragment Library Kit (Thermo Scientific, Wilmington, DE, USA) was used to form the amplicon library according to the manufacturer's instructions, and quantification of the amplicon library was performed using a Bioanalyzer 2100 (Agilent Technologies, Inc., Santa Clara, CA, USA). The amplicon library was sequenced on an Ion Torrent PGM system (Thermo Scientific, Wilmington, DE, USA).

\section{Bioinformatic analysis}

The quantitative insights into microbial ecology (QIIME) pipeline (Caporaso et al. 2010) was used to analyze the sequences. After quality checking the FASTQ file, the barcode sequences of the amplicons were removed. Sequences of 300-440 bp size were filtered and dimers and chimeras were removed, based on Ribosomal Database Project (RDB) database, using ultra-fast sequence analysis (USEARCH 8.1 to ensure high quality. Operational taxonomic units were analyzed based on the Greengenes 13_8 database (McDonald et al. 2012) identified with $97 \%$ similarity. Principal coordinates analysis (PCoA; based on the Bray-Curtis distance) was performed using QIIME 1.9.1. The identification of microbial taxa that were significantly associated with the incubation time was conducted using multivariate association with a linear model (Morgan et al. 2012). Associations with a Benjamini-Hochberg false discovery rate-corrected $p$ value (q value) of $<0.1$ were considered significant. The raw data was uploaded to NCBI sequence read archive database (accession number: DRA006695).

\section{SCFA concentration analysis}

CMRP medium supernatants containing the fecal inoculum of the three donors at each incubation time $(0,6,12$, $18,24,36$, and $48 \mathrm{~h}$ ) were transferred to new tubes, and aliquots were frozen at $-20{ }^{\circ} \mathrm{C}$ for SCFA analysis. Before analysis, samples were filtered through membrane filters (pore size: $0.25 \mu \mathrm{m}$ ). SCFA analysis was performed by ion chromatography (IC) using a 940 Professional IC Vario (Methrohm, Herisau, Switzerland) composed of a two-channel peristaltic pump and a 945 Professional Detector Vario conductivity detector, with an 889 IC Sample Center (Methrohm). IC Net 3.1 software was used to record the data. Ion exclusion was performed on a Metrosep Organic Acid 250/7.8 column (Methrohm) and $0.1 \%$ sulfuric acid was used as the mobile phase, at a flow rate of $0.5 \mathrm{~mL} / \mathrm{min}$ and pressure of $6.99 \mathrm{MPa}$. Samples $(20 \mu \mathrm{L})$ were injected into columns maintained at $30{ }^{\circ} \mathrm{C}$. The peak height, peak area, and retention time of recorded samples and acetic acid, propionic acid, butyric acid, valeric acid, and isovaleric acid standards were used to measure concentrations.

\section{Results}

\section{Pectin degradation by human feces according} to incubation time

Three samples of feces were anaerobically cultured with pectin, and the total carbohydrates in the medium were measured every $6 \mathrm{~h}$ after inoculation (Fig. 1a). Almost all of the carbohydrates in the medium were consumed within $18 \mathrm{~h}$ in all experimental groups. These results suggest that the carbohydrate components in the medium were used by the gut microbiota present in the feces, and particularly, that the pectin was decomposed and used by the gut microbiota. There were differences in the efficiency of carbohydrate usage in each sample. The sample from donor 3 depleted the carbohydrates from the medium more rapidly than the other samples. The amount of reducing sugars was determined for each sample with increasing incubation time (Fig. 1b). The increase in reducing sugar was the highest in donor 3, consistent with the carbohydrate utilization observations. The samples from donors 2 and 3 displayed increased reducing sugars for the first $9 \mathrm{~h}$, followed by decreasing levels thereafter, while the sample from donor 1 showed decreased reducing sugars in the medium from $6 \mathrm{~h}$ onward. This suggests that after the pectin was decomposed by the gut microbiota, increasing the reducing sugar level, the pectin digestion products were consumed, subsequently decreasing the reducing sugar level. The results also suggest that the gut microbiota composition differed between the donors because the ratios of reducing sugar production and consumption varied. Donor 3 might harbor many microorganisms that degrade pectin well, while donor 1 may harbor more microorganisms that utilize pectin degradation products. The carbohydrate contents of the sample media were determined by TLC (Fig. 1c). This confirmed that various fermentable sugars were present in the medium, but most were consumed at 


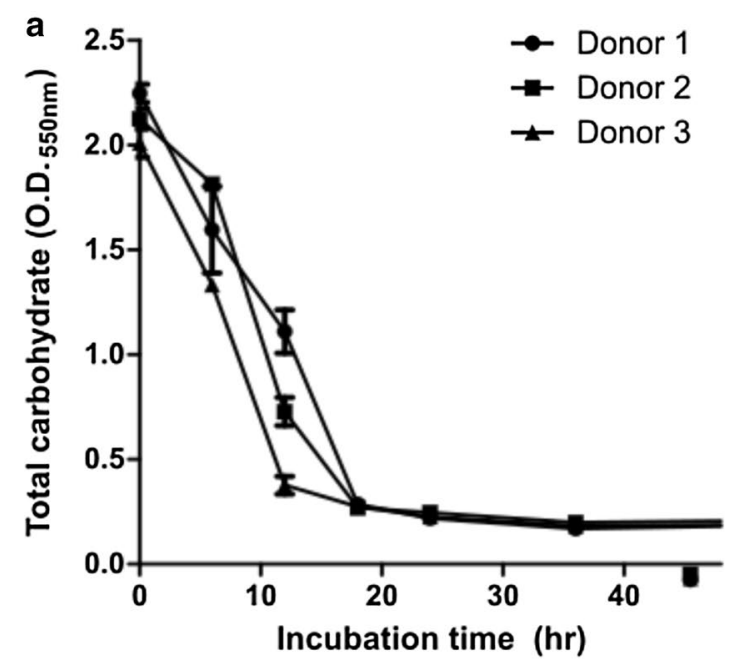

c

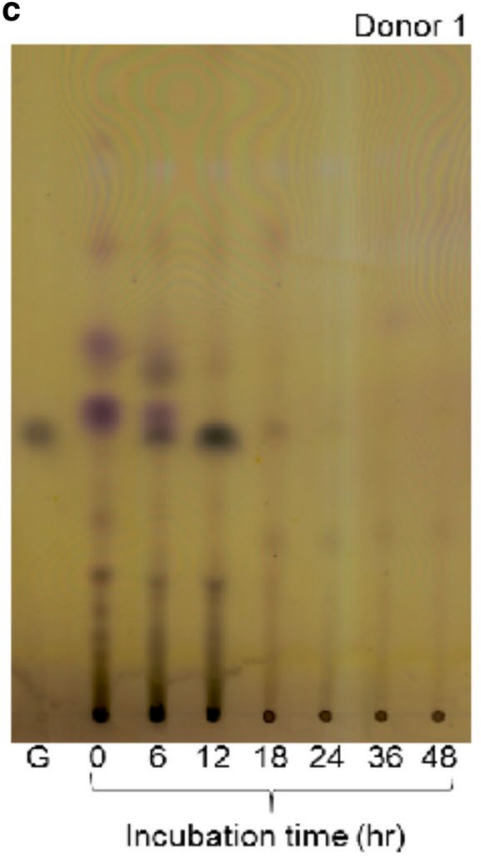

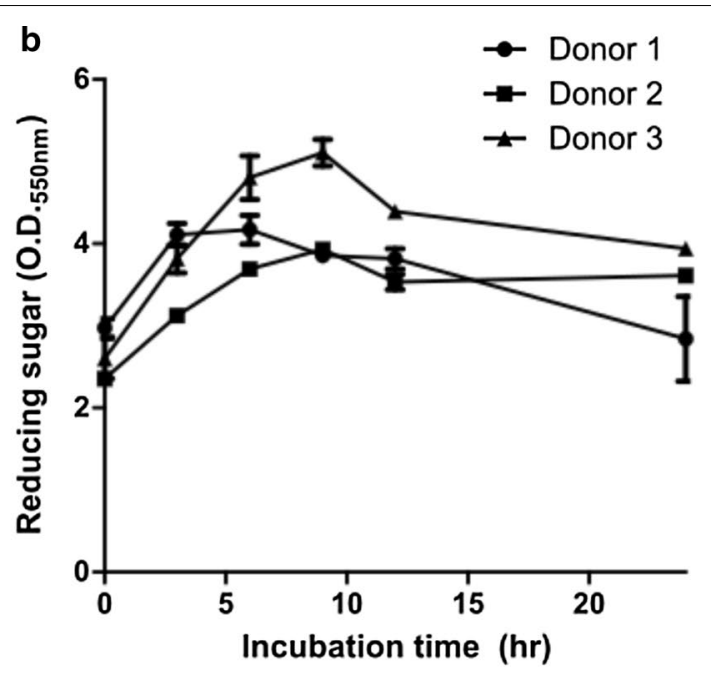

Donor 2

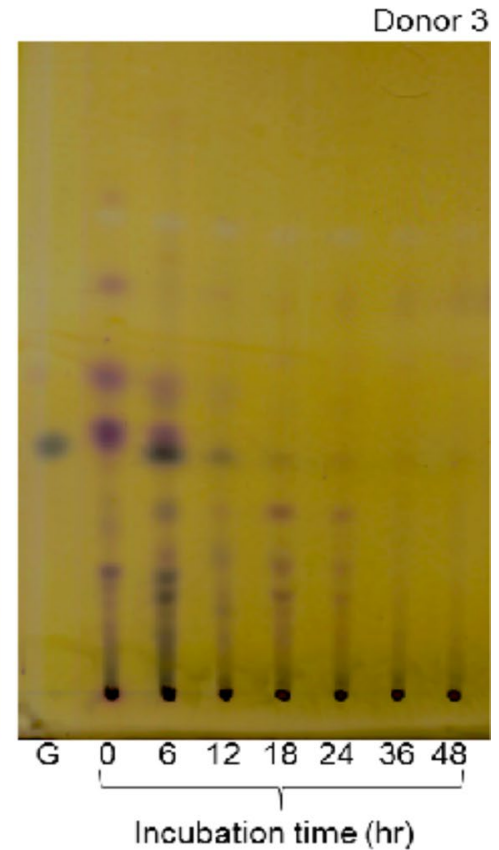

Fig. 1 Total carbohydrates (a), reducing sugars (b), and decomposed pectin products (c) in each donor at various incubation times were analyzed by TLC

early stages. Galacturonic acid, the final degradation product of pectin, was produced after $6-12 \mathrm{~h}$ in all samples and was completely consumed at later time points. In the donor 3 sample, the production of galacturonic acid was faster than in the other samples, which confirmed that more pectin degradation products were produced. These results are consistent with the total carbohydrate and reducing sugar analyses described above, and indicates that the intestinal microbiota differed in each donor.

\section{Fecal microbial composition of each donor at baseline}

To investigate the in vitro fermentation of pectin by the human fecal microbiota, we anaerobically incubated the feces of the three donors with $1 \%$ pectin for $18 \mathrm{~h}$. The compositions of the fecal microbiota samples were observed at baseline (in raw fecal samples which were not mixed with medium or pectin) and after every $6 \mathrm{~h}$ of incubation time using $16 \mathrm{~S}$ rRNA gene sequencing.

We first examined the initial fecal microbiota compositions of each donor. At the phylum level, Firmicutes 
(57.39\% of average relative abundance) and Bacteroidetes $(42.11 \%)$ were dominant in all three donors, and Proteobacteria (0.26\%) and Actinobacteria (0.02\%) were also present in small proportions (Fig. 2a). The Firmicutes/Bacteroidetes ratios were higher in donor 1 (2.21 ratio) and donor 3 (1.52 ratio) compared to donor 2 (0.78 ratio). We next examined the top 20 most abundant genera in each baseline sample (Fig. 2b). Donors 1, 2, and 3 had 18, 12, and 11 genera comprising $>1 \%$ relative abundance, respectively. In all three donors, Bacteroides and Ruminococcaceae were the most abundant. The relative abundance of Bacteroides in donor 2 was much higher (46.34\%) than that in donors 1 and 3 (19.20 and $37.39 \%$, respectively). In addition, each donor displayed differences in bacterial abundance. For example, donor 3 harbored more Lachnospiraceae and Faecalibacterium than the others, while donor 1 and donor 2 harbored more Streptococcus, unclassified Rikenellaceae, Lactobacillus, Lachnospiraceae, and Prevotella.

\section{Changes in fecal microbial composition during pectin fermentation}

To evaluate the influence of pectin fermentation on the overall structure of the fecal microbiota, we conducted PCoA based on Bray-Curtis distances. Samples from each donor clustered separately from those of the other donors, showing that the donor had the greatest effect on microbiome composition, even after incubation with pectin for $18 \mathrm{~h}$ (Fig. 3). However, among samples from each donor, the microbial composition changed with increased incubation time. For donor 3, for example, the

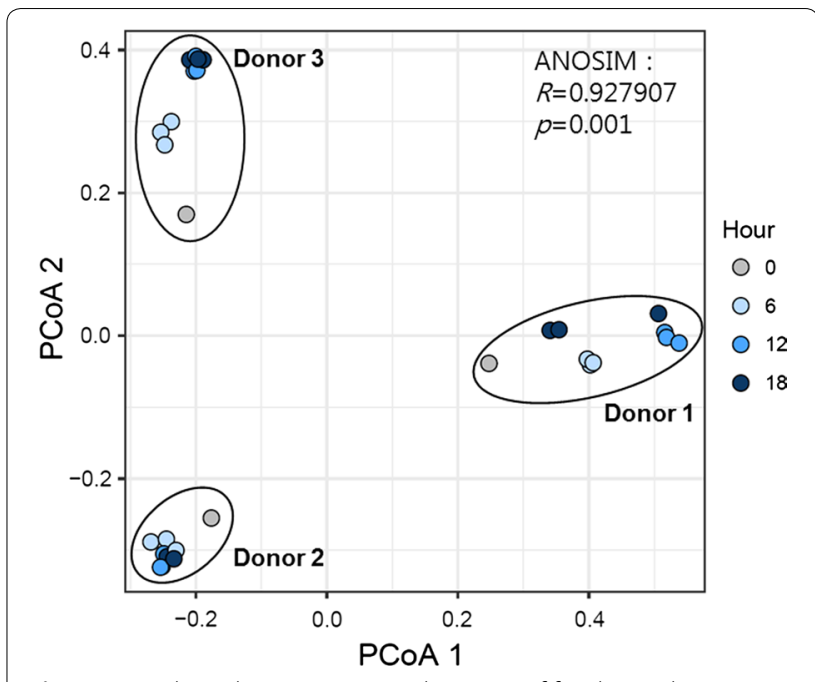

Fig. 3 PCoA based on Bray-Curtis distances of fecal samples incubated with pectin for various incubation times

baseline sample was most similar to the sample after $6 \mathrm{~h}$ pectin incubation, followed by those taken at 12 and $18 \mathrm{~h}$.

To identify microbes whose abundances significantly changed with increased incubation time, we explored the associations between bacterial abundance and incubation time by linear regression analysis. The levels of several specific bacteria were associated with time. For example, Lachnospira, Sutterella, Dorea, and Clostridium were significantly increased over time, while Bacteroides and Roseburia were significantly decreased (Fig. 4 and Additional file 1: Figure S1).

\section{a Phylum}

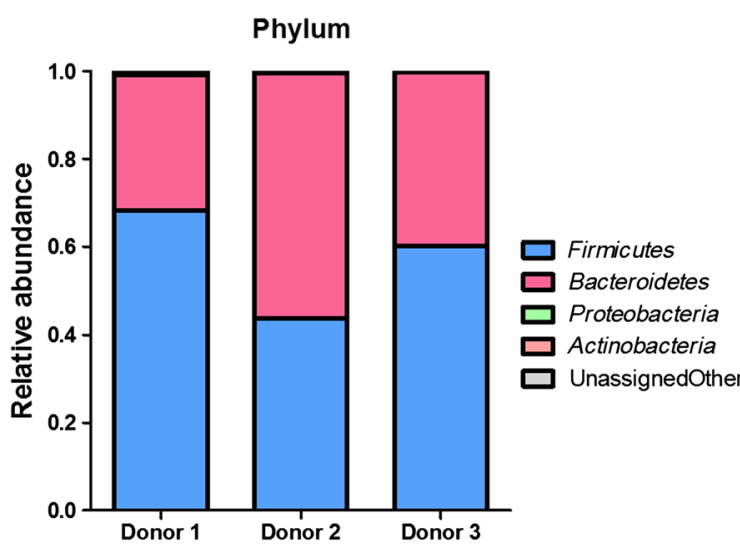

\section{b Genus (average abundance top 20)}

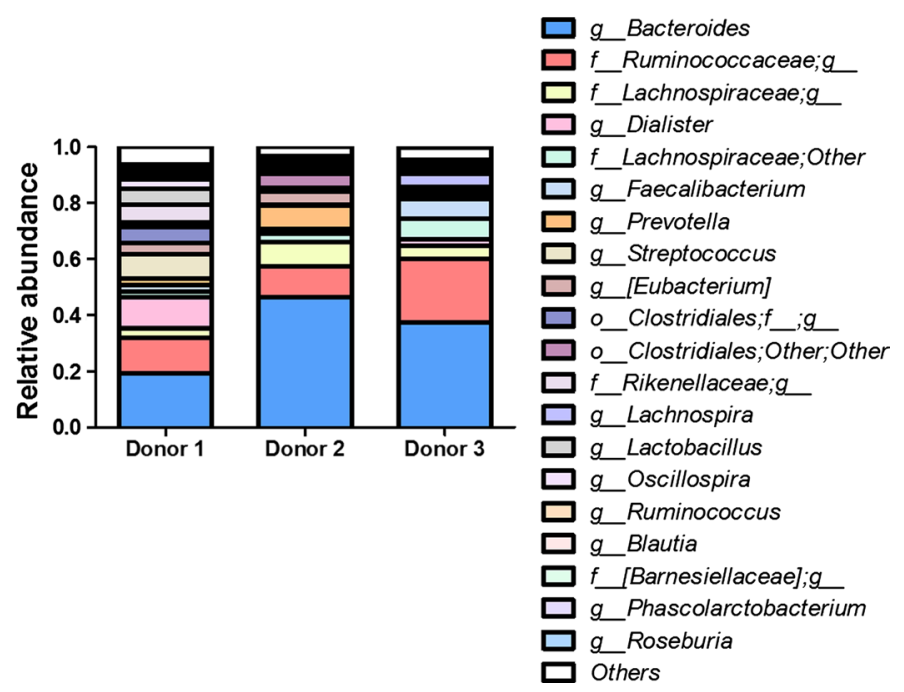

Fig. 2 Baseline fecal microbial compositions of each donor, including the relative abundance of phyla (a) and the 20 most abundant genera (b) 


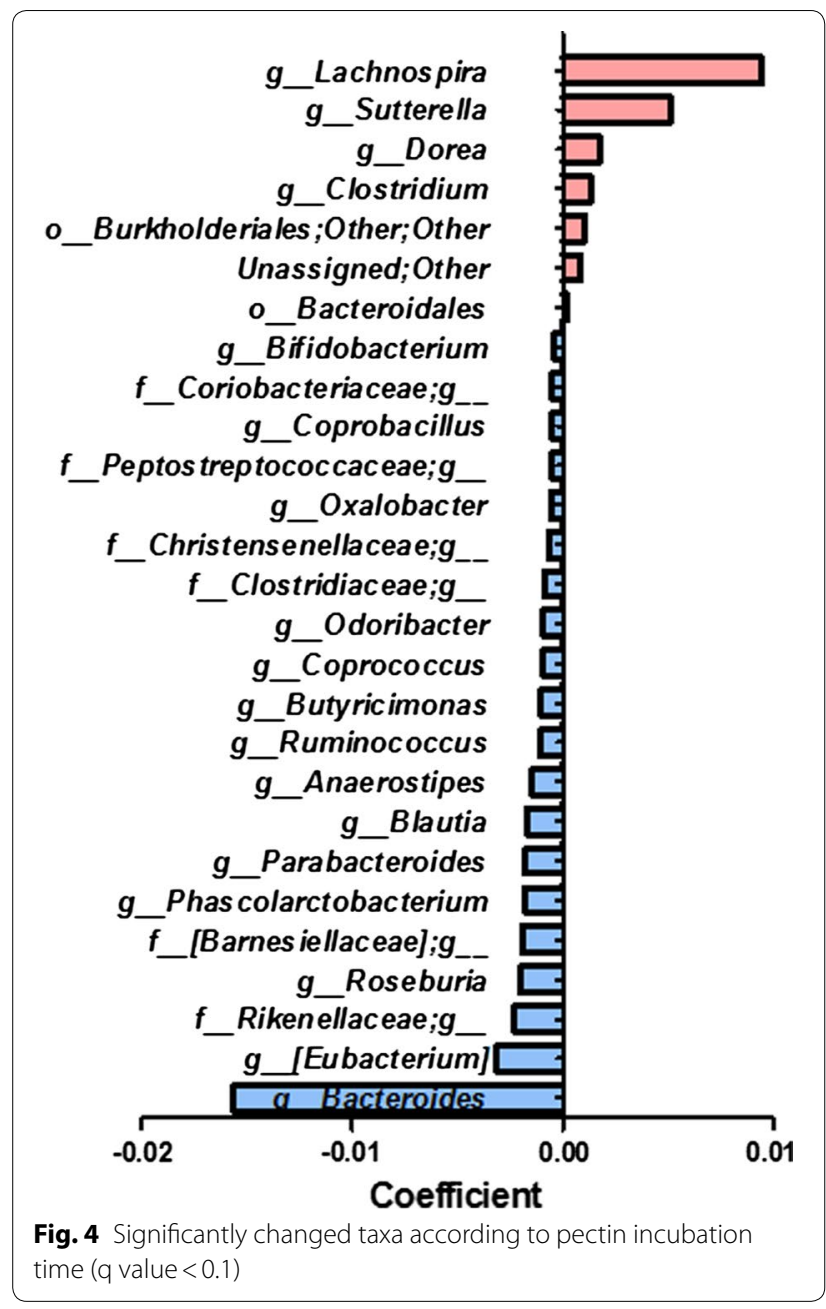

SCFA formation during pectin fermentation

When we analyzed the SCFA changes in each sample every $6 \mathrm{~h}$, we observed rapid increases in acetate after $6 \mathrm{~h}$, which continuously increased up to $18 \mathrm{~h}$, then rapidly decreased by $36 \mathrm{~h}$. Propionate increased after $48 \mathrm{~h}$, and while butyrate did not rapidly increase like acetate, it increased by approximately $28 \%$ by $48 \mathrm{~h}$ (Fig. 5 ).

\section{Discussion}

The contributions of the gut microbiome to health and nutrition depend on its composition, which is affected by different factors, including lifestyle and diet (Conlon and Bird 2014). Gut microbiota composition can be changed by including indigestible carbohydrates (prebiotics) in one's diet (Flint et al. 2012). Pectin is a prebiotic dietary fiber that affects the gut microbiota (Woods and Gorbach 2001). In this study, we investigated the utilization of pectin by the gut microbiota and analyzed microbiota composition changes with in vitro pectin fermentation through metagenomics analysis.

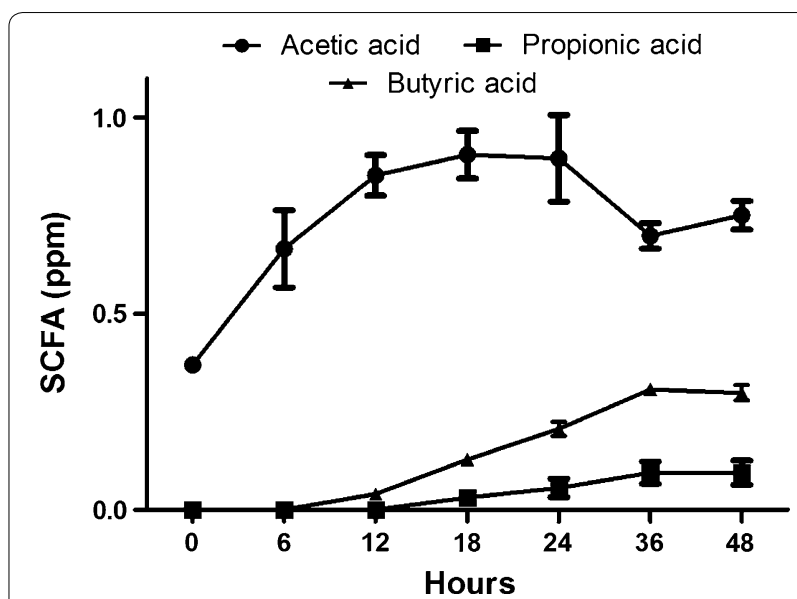

Fig. 5 Average SCFA concentrations at various pectin incubation times

Pectin was mainly degraded between 0 and $18 \mathrm{~h}$ in all three donors, but the samples showed differences in their pectin degradation ability. These differences in substrate utility depend on the composition of the microbiome (Flint et al. 2008). When pectin was digested, galacturonic acid is produced (De Vries et al. 1982). Monosaccharide such as galacturonic acid is used as an energy source by bacteria and contributes to the development and maintenance of the gut microbiota (Zoetendal et al. 2012). In this study, the microbiota of donor 3 showed relatively high pectin utilization, and as expected, this sample contained higher baseline Lachnospiraceae and Faecalibacterium levels than in the other samples. Lachnospiraceae has been demonstrated to express carbohydrate-active and pectin-degrading enzymes (Biddle et al. 2013), and Faecalibacterium also has reported pectin degradation ability (Lopez-Siles et al. 2012). In addition, the sample from donor 2 consumed more galacturonic acid than the others once the reducing sugar level increased after $6 \mathrm{~h}$. These results indicate that differences in the baseline levels of different microbiome components result in differences in pectin degradation and utilization in each donor.

We observed comprehensive overall gut microbiota changes after pectin fermentation in all three donors compared to their baseline compositions. We not only observed differences between each donor's gut microbiota, but also changes with increased incubation time by PCoA. We assumed that specific gut microbiota were utilizing pectin as a substrate; for example, Prevotella and Butyrivibrio spp., which express pectinolytic enzymes (Marounek and Dušková 1999). With increased incubation time with pectin, we observed increases in Lachnospira, Sutterella, Dorea, and Clostridium. The 
Clostridium coccoides group (cluster XIVa) includes Lachnospira, Dorea, and Clostridium (Lopetuso et al. 2013). Lachnospira was the most increased and has been reported to use pectin as a substrate (Wojciechowicz et al. 1980) and Lachnospira plays a role that produced SCFA (Duvallet et al. 2017; Jones et al. 2014). Pectin degradation by Lachnospira affects the growth of other bacteria, including other saccharolytic bacteria, via crossfeeding (Salyers and Leedle 1983). Increases in Dorea and Clostridium may be due to Lachnospira cross-feeding. These results indicate that pectin promotes the presence of species in Clostridium cluster XIV, including Lachnospira, Dorea, and Clostridium. We also observed that some bacteria, including Bacteroides, which can utilize pectin (Gibson and Roberfroid 1995), decreased with increasing pectin incubation time, possibly due to environmental nutritional limitations caused by selective culture with pectin and carbohydrate consumption.

The production of SCFAs, including acetate, propionate, and butyrate, is affected by the composition of the gut microbiota, the utilization of carbohydrate substrates, and the gut environment, including the $\mathrm{pH}$ and other nutritional factors (Cummings and Englyst 1987; Yuan et al. 2006). Walker et al. (2005) reported that the production acetate and propionate increased at $\mathrm{pH} 6.5$. Lachnospira produces the most pectin lyase at $\mathrm{pH} 6.1-$ 6.3 (Silley 1986), and in pectin culture, mainly produces acetate and lactate (Dušková and Marounek 2001). The Clostridium genus is known to mainly produce butyrate (Rajilić-Stojanović and de Vos 2014). Based on this, we suggest that Lachnospira produced galacturonic acid through the digestion of pectin and acetate, and that this environment restrained the growth of Bacteroides by causing a mildly acidic $\mathrm{pH}$. Produced galacturonic acid affected the composition of the gut microbiota, enhancing cluster XIVa species belonging to Dorea and Clostridium, which then produced butyrate (Duncan et al. 2007; Walker et al. 2005). We observed decreased acetate after $18 \mathrm{~h}$ of pectin incubation, indicating that not only galacturonic acid but also acetate was utilized by bacteria like Faecalibacterium to produce butyrate (Duncan et al. 2004; Ramirez-Farias et al. 2008).

This result suggests that acetate-producing bacteria like Lachnospira and Faecalibacterium caused increased butyrate levels via butyrate synthesis using acetate as a substrate (Khan et al. 2012; Rios-Covian et al. 2015). Based on this, it appears that pectin degradation results in a gut microbiota growth environment associated with the development of acetate and butyrate.

In conclusion, we demonstrate that in Korean individuals, pectin can change the gut microbiota by measuring total sugar levels and microbial composition over time. Pectin was completely degraded by the gut microbiota at 6,12, and $18 \mathrm{~h}$, and Lachnospira and Faecalibacterium, which can utilize pectin, were increased. Pectin-induced changes in the gut microbiota increased the formation of associated SCFAs from $6 \mathrm{~h}$ on, when pectin was decomposed. Therefore, we confirmed that pectin fermentation in gut microbiota samples from Korean individuals induced microbiota compositional changes. Increased pectin utilization and corresponding changes to gut microbiome composition may be beneficial to human health. Further analysis of the gut microbiomes of larger numbers of donors, in addition to experiments regarding cross-feeding and in vivo gut microbiota changes, would provide more accurate results.

\section{Additional file}

Additional file 1: Figure S1. Several bacterial taxa significantly associated with pectin incubation time. Each donor is color-coded as indicated by the legend; (A) Lachnospira, (B) Roseburia, and (C) Bacteroides.

\section{Abbreviations \\ SCFAs: short chain fatty acids; CMR: bovine rumen fluid; CMRP: bovine rumen fluid pectin; DNS: 3,5-dinitrosalicylic acid; TLC: thin liquid chromatography; PCR: polymerase chain reaction; QIIME: the quantitative insights into microbial ecology; PCoA: principal coordinates analysis; IC: ion chromatography; USE- ARCH: ultra-fast sequence analysis; RDP: Ribosomal Database Project.}

\section{Authors' contributions}

C-SP and D-HS designed and coordinated all the experiments; S-JB performed the experiments and analyzed the gut microbiota data; GK conducted experiments on pectin degradation of human fecal microbiome; MYL, E-JS, and $Y$-DN performed statistical analysis and metagenome analysis of human fecal microbial change; D-HJ performed anaerobic cultivation of the human fecal microbiome; J-SK confirmed the SCFA production by ion chromatography analysis. S-JB, GK, C-SP, and D-HS prepared the manuscript, evaluated the results and revised the manuscript. All authors read and approved the final manuscript.

\section{Author details \\ ${ }^{1}$ Graduate School of Biotechnology and Institute of Life Science and Resources, Kyung Hee University, Yongin 17104, Republic of Korea. ${ }^{2}$ Research Group of Healthcare, Korea Food Research Institute, Wanju 55365, Republic of Korea. ${ }^{3}$ Department of Food Biotechnology, Korea University of Science and Technology, Daejeon 34113, Republic of Korea. ${ }^{4}$ Research Group of Food Processing, Korea Food Research Institute, Wanju 55365, Republic of Korea.}

\section{Competing interests}

The authors declare that they have no competing interests.

\section{Availability of data and materials}

Not applicable.

\section{Consent for publication \\ Not applicable.}

\section{Ethics approval and consent to participate}

All procedures performed in studies involving human participants were in accordance with the ethical standards of the institutional and/or national research committee and with the 1964 Helsinki declaration and its later amendments or comparable ethical standards. 


\section{Funding}

This research was supported by the Main Research Program (E0170602-02) of the Korea Food Research Institute funded by the Ministry of Science and ICT for Dr. Y-D Nam and was supported by a National Research Foundation of Korea (NRF) Grant funded by the Korean government (MEST) (No. 2017R1A2B4004218) for Dr. C-S Park.

\section{Publisher's Note}

Springer Nature remains neutral with regard to jurisdictional claims in published maps and institutional affiliations.

Received: 30 May 2018 Accepted: 12 June 2018

Published online: 16 June 2018

\section{References}

Biddle A, Stewart L, Blanchard J, Leschine S (2013) Untangling the genetic basis of fibrolytic specialization by Lachnospiraceae and Ruminococcaceae in diverse gut communities. Diversity 5(4):627-640. https://doi. org/10.3390/d5030627

Bindels LB, Delzenne NM, Cani PD, Walter J (2015) Towards a more comprehensive concept for prebiotics. Nat Rev Gastroenterol Hepatol 12(5):303-310. https://doi.org/10.1038/nrgastro.2015.47

Caporaso JG, Kuczynski J, Stombaugh J, Bittinger K, Bushman FD, Costello EK, Fierer N, Pena AG, Goodrich JK, Gordon JI (2010) QIIME allows analysis of high-throughput community sequencing data. Nat Methods 7(5):335

Chung WS, Walker AW, Louis P, Parkhill J, Vermeiren J, Bosscher D, Duncan SH, Flint HJ (2016) Modulation of the human gut microbiota by dietary fibres occurs at the species level. BMC Biol 14:3. https://doi.org/10.1186/s1291 5-015-0224-3

Clemente JC, Ursell LK, Parfrey LW, Knight R (2012) The impact of the gut microbiota on human health: an integrative view. Cell 148(6):1258-1270. https://doi.org/10.1016/j.cell.2012.01.035

Conlon MA, Bird AR (2014) The impact of diet and lifestyle on gut microbiota and human health. Nutrients 7(1):17-44. https://doi.org/10.3390/nu701 0017

Coppa GV, Zampini L, Galeazzi T, Gabrielli O (2006) Prebiotics in human milk: a review. Dig Liver Dis 38:S291-S294. https://doi.org/10.1016/s1590 -8658(07)60013-9

Cummings JH, Englyst HN (1987) Fermentation in the human large intestine and the available substrates. Am J Clin Nutr 45(5):1243-1255

David LA, Maurice CF, Carmody RN, Gootenberg DB, Button JE, Wolfe BE, Ling AV, Devlin AS, Varma Y, Fischbach MA, Biddinger SB, Dutton RJ, Turnbaugh PJ (2014) Diet rapidly and reproducibly alters the human gut microbiome. Nature 505(7484):559-563. https://doi.org/10.1038/nature12820

De Vries J, Rombouts F, Voragen A, Pilnik W (1982) Enzymic degradation of apple pectins. Carbohydr Polym 2(1):25-33

Duncan SH, Holtrop G, Lobley GE, Calder AG, Stewart CS, Flint HJ (2004) Contribution of acetate to butyrate formation by human faecal bacteria. $\mathrm{Br} J$ Nutr 91(6):915-923. https://doi.org/10.1079/BJN20041150

Duncan SH, Belenguer A, Holtrop G, Johnstone AM, Flint HJ, Lobley GE (2007) Reduced dietary intake of carbohydrates by obese subjects results in decreased concentrations of butyrate and butyrate-producing bacteria in feces. Appl Environ Microbiol 73(4):1073-1078. https://doi.org/10.1128/ AEM.02340-06

Dušková D, Marounek M (2001) Fermentation of pectin and glucose, and activity of pectin-degrading enzymes in the rumen bacterium Lachnospira multiparus. Lett Appl Microbiol 33(2):159-163

Duvallet C, Gibbons SM, Gurry T, Irizarry RA, Alm EJ (2017) Meta analysis of microbiome studies identifies shared and disease-specific patterns. Nat Commun 8(1):1784. https://doi.org/10.1038/s41467-017-01973-8

Falony G, Joossens M, Vieira-Silva S, Wang J, Darzi Y, Faust K, Kurilshikov A, Bonder MJ, Valles-Colomer M, Vandeputte D, Tito RY, Chaffron S, Rymenans L, Verspecht C, De Sutter L, Lima-Mendez G, D'hoe K, Jonckheere K, Homola D, Garcia R, Tigchelaar EF, Eeckhaudt L, Fu J, Henckaerts L, Zhernakova A, Wijmenga C, Raes J (2016) Population-level analysis of gut microbiome variation. Science 352(6285):560-564. https://doi. org/10.1126/science.aad3503
Flint HJ, Bayer EA, Rincon MT, Lamed R, White BA (2008) Polysaccharide utilization by gut bacteria: potential for new insights from genomic analysis. Nat Rev Microbiol 6(2):121-131. https://doi.org/10.1038/nrmicro1817

Flint HJ, Scott KP, Louis P, Duncan SH (2012) The role of the gut microbiota in nutrition and health. Nat Rev Gastroenterol Hepatol 9(10):577-589. https ://doi.org/10.1038/nrgastro.2012.156

Gerritsen J, Smidt H, Rijkers GT, de Vos WM (2011) Intestinal microbiota in human health and disease: the impact of probiotics. Genes Nutr 6(3):209-240. https://doi.org/10.1007/s12263-011-0229-7

Gevers D, Kugathasan S, Denson LA, Vazquez-Baeza Y, Van Treuren W, Ren B, Schwager E, Knights D, Song SJ, Yassour M, Morgan XC, Kostic AD, Luo C, Gonzalez A, McDonald D, Haberman Y, Walters T, Baker S, Rosh J, Stephens M, Heyman M, Markowitz J, Baldassano R, Griffiths A, Sylvester F, Mack D, Kim S, Crandall W, Hyams J, Huttenhower C, Knight R, Xavier RJ (2014) The treatment-naive microbiome in new-onset Crohn's disease. Cell Host Microbe 15(3):382-392. https://doi.org/10.1016/j.chom.2014.02.005

Gibson GR, Roberfroid MB (1995) Dietary modulation of the human colonic microbiota: introducing the concept of prebiotics. J Nutr 125(6):1401-1412

Holloway WD, Tasman-Jones C, Maher K (1983) Pectin digestion in humans. Am J Clin Nutr 37(2):253-255

Hyland NP, Quigley EM, Brint E (2014) Microbiota-host interactions in irritable bowel syndrome: epithelial barrier, immune regulation and brain-gut interactions. World J Gastroenterol 20(27):8859-8866. https://doi. org/10.3748/wjg.v20.i27.8859

Icaza-Chávez M (2013) Gut microbiota in health and disease. Rev Gastroenterol Mex 78(4):240-248

Jones ML, Ganopolsky JG, Martoni CJ, Labbé A, Prakash S (2014) Emerging science of the human microbiome. Gut Microbes 5(4):446-457. https:// doi.org/10.4161/gmic.29810

Khan MT, Duncan SH, Stams AJ, van Dijl JM, Flint HJ, Harmsen HJ (2012) The gut anaerobe Faecalibacterium prausnitzii uses an extracellular electron shuttle to grow at oxic-anoxic interphases. ISME J 6(8):1578-1585. https:// doi.org/10.1038/ismej.2012.5

Kim SH, Kim MS, Lee MS, Park YS, Lee HJ, SA Kang, Lee HS, Lee KE, Yang HJ, Kim MJ, Lee YE, Kwon DY (2016) Korean diet: characteristics and historical background. J Ethnic Foods 3(1):26-31. https://doi.org/10.1016/j. jef.2016.03.002

Laparra JM, Sanz Y (2010) Interactions of gut microbiota with functional food components and nutraceuticals. Pharmacol Res 61(3):219-225. https:// doi.org/10.1016/j.phrs.2009.11.001

LeCroy MN, Stevens J (2017) Dietary intake and habits of South Asian immigrants living in western countries. Nutr Rev 75(6):391-404. https://doi. org/10.1093/nutrit/nux023

Lee MJ, Popkin BM, Kim S (2002) The unique aspects of the nutrition transition in South Korea: the retention of healthful elements in their traditional diet. Public Health Nutr 5(1A):197-203. https://doi.org/10.1079/PHN20 01294

Li J, Hou Q, Zhang J, Xu H, Sun Z, Menghe B, Zhang H (2017) Carbohydrate staple food modulates gut microbiota of mongolians in China. Front Microbiol 8:484. https://doi.org/10.3389/fmicb.2017.00484

Lopetuso LR, Scaldaferri F, Petito V, Gasbarrini A (2013) Commensal Clostridia: leading players in the maintenance of gut homeostasis. Gut Pathog 5(1):23

Lopez-Siles M, Khan TM, Duncan SH, Harmsen HJ, Garcia-Gil LJ, Flint HJ (2012) Cultured representatives of two major phylogroups of human colonic Faecalibacterium prausnitzii can utilize pectin, uronic acids, and hostderived substrates for growth. Appl Environ Microbiol 78(2):420-428. https://doi.org/10.1128/AEM.06858-11

Marounek M, Dušková D (1999) Metabolism of pectin in rumen bacteria Butyrivibrio fibrisolvens and Prevotella ruminicola. Lett Appl Microbiol 29(6):429-433. https://doi.org/10.1046/j.1472-765X.1999.00671.x

Marques TM, Wall R, Ross RP, Fitzgerald GF, Ryan CA, Stanton C (2010) Programming infant gut microbiota: influence of dietary and environmental factors. Curr Opin Biotechnol 21(2):149-156. https://doi.org/10.1016/j. copbio.2010.03.020

McDonald D, Price MN, Goodrich J, Nawrocki EP, DeSantis TZ, Probst A, Andersen GL, Knight R, Hugenholtz P (2012) An improved Greengenes taxonomy with explicit ranks for ecological and evolutionary analyses of bacteria and archaea. ISME J 6(3):610 
Morgan XC, Tickle TL, Sokol H, Gevers D, Devaney KL, Ward DV, Reyes JA, Shah SA, LeLeiko N, Snapper SB, Bousvaros A, Korzenik J, Sands BE, Xavier RJ, Huttenhower C (2012) Dysfunction of the intestinal microbiome in inflammatory bowel disease and treatment. Genome Biol 13(9):R79. https ://doi.org/10.1186/gb-2012-13-9-r79

Rajilić-Stojanović M, de Vos WM (2014) The first 1000 cultured species of the human gastrointestinal microbiota. FEMS Microbiol Rev 38(5):996-1047. https://doi.org/10.1111/1574-6976.12075

Ramirez-Farias C, Slezak K, Fuller Z, Duncan A, Holtrop G, Louis P (2008) Effect of inulin on the human gut microbiota: stimulation of Bifidobacterium adolescentis and Faecalibacterium prausnitzii. Br J Nutr 101(4):541-550. https://doi.org/10.1017/S0007114508019880

Rios-Covian D, Gueimonde M, Duncan SH, Flint HJ, de los Reyes-Gavilan CG (2015) Enhanced butyrate formation by cross-feeding between Faecalibacterium prausnitzii and Bifidobacterium adolescentis. FEMS Microbiol Lett. https://doi.org/10.1093/femsle/fnv176

Salyers AA, Leedle JAZ (1983) Carbohydrate metabolism in the human colon. In: Hentges DJ (ed) Human intestinal microflora in health and disease. Academic Press, London, pp 129-146

Sánchez B, Delgado S, Blanco-Miguez A, Lourenço A, Gueimonde M, Margolles A (2017) Probiotics, gut microbiota, and their influence on host health and disease. Mol Nutr Food Res. https://doi.org/10.1002/mnfr.201600240

Silley P (1986) The production and properties of a crude pectin lyase from Lachnospira multiparas. Lett Appl Microbiol 2(2):29-31. https://doi. org/10.1111/j.1472-765X.1986.tb01509.x
Song Y, Joung H (2012) A traditional Korean dietary pattern and metabolic syndrome abnormalities. Nutr Metab Cardiovasc Dis 22(5):456-462. https ://doi.org/10.1016/j.numecd.2010.09.002

Sriamornsak P (2003) Chemistry of pectin and its pharmaceutical uses: a review. Silpakorn Univ Int J 3(1-2):206-228

Walker AW, Duncan SH, McWilliam Leitch EC, Child MW, Flint HJ (2005) pH and peptide supply can radically alter bacterial populations and shortchain fatty acid ratios within microbial communities from the human colon. Appl Environ Microbiol 71(7):3692-3700. https://doi.org/10.1128/ AEM.71.7.3692-3700.2005

Wojciechowicz M, Heinrichova K, Ziołecki A (1980) A polygalacturonate lyase produced by Lachnospira multiparus isolated from the bovine rumen. J Gen Microbiol 117(1):193-199. https://doi.org/10.1099/00221 287-117-1-193

Woods MN, Gorbach SL (2001) Influences of fibers on the ecology of the intestinal flora. In: Spiller GA (ed) Handbook of dietary fibre in human nutrition. CRC, New York, pp 257-270

Yuan H, Chen Y, Zhang H, Jiang S, Zhou Q, Gu G (2006) Improved bioproduction of short-chain fatty acids (SCFAs) from excess sludge under alkaline conditions. Environ Sci Technol 40(6):2025-2029

Zoetendal EG, Raes J, van den Bogert B, Arumugam M, Booijink CC, Troost FJ, Bork P, Wels M, de Vos WM, Kleerebezem M (2012) The human small intestinal microbiota is driven by rapid uptake and conversion of simple carbohydrates. ISME J 6(7):1415-1426. https://doi.org/10.1038/ismej .2011 .212

\section{Submit your manuscript to a SpringerOpen ${ }^{\circ}$ journal and benefit from:}

- Convenient online submission

Rigorous peer review

- Open access: articles freely available online

- High visibility within the field

- Retaining the copyright to your article

Submit your next manuscript at springeropen.com 